\title{
Effect of melatonin addition in ovulation induction protocols with clomiphene citrate in management of infertility
}

\author{
Anjali Chaudhary, Aditi Agarwal*, Meenakshi Tanwar, , Parul Singh, Priyanka Negi
}

Department of Obstetrics and Gynecology, Sri Guru Ram Rai Institute of Medical and Health Science, Dehradun, Uttarakhand, India

Received: 01 September 2021

Revised: 17 November 2021

Accepted: 18 November 2021

*Correspondence:

Dr. Aditi Agarwal,

E-mail: aditiagarwal47@gmail.com

Copyright: () the author(s), publisher and licensee Medip Academy. This is an open-access article distributed under the terms of the Creative Commons Attribution Non-Commercial License, which permits unrestricted non-commercial use, distribution, and reproduction in any medium, provided the original work is properly cited.

\section{ABSTRACT}

Background: About $10-12 \%$ of couples are unable to conceive by natural means despite concerted efforts and these figures have risen in past few decades. In recent years, effect of oxidative stress on the fertility has been widely recognised. Oxidative stress has been known to affect both male and female fertility reducing sperm count and motility in men, and affecting ovum reserve and quality in women. Melatonin is the secretion of the pineal gland responsible for circadian sleep rhythm has also shown to be good antioxidant. Aim and objective of current study was to study effect of melatonin addition to clomiphene citrate for induction of ovulation with a view to improve conception rates.

Methods: This was a retrospective analysis of 52 women with infertility who were given clomiphene citrate with melatonin. Ovulation, conception rates and pregnancy outcomes were noted.

Results: We observed $77.8 \%$ ovulation rates, and a significantly better conception and live birth rates.

Conclusions: Melatonin, as an anti-oxidant may improve, conception and live birth rates when added to clomiphene citrate induction protocols.

Keywords: Infertility, Antioxidants, Melatonin, Ovulation induction, Clomiphene citrate

\section{INTRODUCTION}

Although, infertility management in recent times has become easier thanks to proliferation of ART centres, but the stress of treatment and societal stigma of childlessness still prevails. About 10-12 \% of couples are unable to conceive by natural means despite concerted efforts and these figures have risen in past few decades. Postponement of parenthood for academic or career prospects, lifestyle and environmental changes, increased incidence of genital infections, misuse of medical abortion, endometriosis and declining ovarian reserve are some of the causes related to the rise in subfertility. In about $3-37 \%$ women infertility is said to be unexplained where there is inability to conceive in spite of ovulation, normal semen parameters and patent tubes.

In recent years, effect of oxidative stress on the fertility has been widely recognised. ${ }^{1}$ Oxidative stress has been known to affect both male and female fertility reducing sperm count and motility in men, and affecting ovum reserve and quality in women. Excessive intracellular production of reactive oxygen species (ROS) or the metabolites of oxidation can damage sperm and oocyte quality pathologically. ${ }^{2}$ Body produces antioxidant compounds as a natural defence against these ROS, but when natural antioxidants levels fall and ROS rise, causing an imbalance which leads to loss of protective 
effect of antioxidants and impairment of reproductive performance results.

An antioxidant is any substance that at low concentration delays the oxidation of proteins, carbohydrates, lipids and DNA, oxidation produces free radicles responsible for the chain reaction that causes cell damage. ${ }^{3}$ There are three categories of antioxidants naturally present in humans viz. vitamins (vitamin $\mathrm{A}, \mathrm{C}, \mathrm{E}$ ), enzymes and trace elements like Selenium, copper and zinc. Certain other substance like Q10, lycopene also helps in clearing free radicles from cells and improve physiological function.

Melatonin is the secretion of the pineal gland responsible for circadian sleep rhythm has also shown to be good antioxidant. Melatonin (N-acetyl-5-methoxytryptamine) is secreted during the dark hours at night, and it regulates a variety of important central and peripheral actions related to circadian rhythms and reproduction. ${ }^{4}$ Melatonin may be a key factor in the control of seasonal changes in gonadal activity. The circadian disturbances related to reproduction are probably subsequent to the seasonal change. Moreover, melatonin might also be considered essential for both spermatogenesis and folliculogenesis. ${ }^{5}$

Melatonin exerts its effect on reproduction by regulating the gonadotrophic secretions in hypothalamo-pituitary axis through certain receptors in addition it also believed to be great of help to free intracellular radicles, and help decrease cellular injury. In contrast to the majority of other known radical scavengers, melatonin is multifunctional and a universal antioxidant, high quantities of melatonin have been found in follicular fluid. $^{6}$ Melatonin is a very potent and efficient endogenous radical scavenger. This pineal indolamine reacts with the highly toxic hydroxyl radical and provides on-site protection against oxidative damage to biomolecules within every cellular compartment. ${ }^{7}$ The antioxidants act by donating electrons to stabilize toxic free radicals. $^{8}$ All reproductive processes like mitosis, meiosis, chromosome segregation, fertilization etc require energy and at the cellular level these processes carry on smoothly if the intracellular fluid is not burdened with excess of toxic ROS. ${ }^{9}$

Melatonin added to the ovulation induction protocols in ART can help decrease the oxidative stress and improve sperm and ovum quality, thereby improving the success of treatment. Generally, women seeking infertility treatment are older and are expected to have higher concentration of ROS and oxidative stress resulting in mitochondrial damage and decreased ATP production. This has a direct effect on quality of oocyte ,embryo and the outcome of ART procedure. ${ }^{10}$ Shavi et al in a double blind pilot study of effect on melatonin on infertility treatment found that the melatonin in ART (MIART) trial has the potential to improve IVF treatment protocols, with both immediate and translational benefit to patients, and should be a routine part of management of infertile couple. ${ }^{9}$ Melatonin is also been found useful in an infertility, there is an increasing evidence that it protects against age related mitochondrial oxidative damage. Chio song and colleagues in their study on mice conclude that long term melatonin treatment for 12 months significantly reduced ovarian aging, and improved oocyte quantity and quality. Treatment with melatonin suppresses mitochondrial oxidative damage in ovary by reducing mitochondrial reactive oxygen species (MROS) preserving the respiratory chain and decreasing oocyte apoptosis. $^{11}$

The most challenging aspect of ART protocols is ovulation induction. A well stimulated cycle is the one with adequate ovarian and endometrial response which would lead to successful ovulation and conception. Clomiphene citrate is the oldest safe and effective and time-tested agent used for ovulation induction protocols either alone or in conjunction with gonadotrophins in an adequately stimulated cycle a $60-80 \%$ ovulation and 30 $40 \%$ conception rate is expected. ${ }^{12}$ Seyedoshohadaei et al in a comparative study of clomiphene citrate, tamoxifen and letrozole for ovulation induction observed higher pregnancy rate with clomiphene citrate than tamoxifen and letrozole, clomiphene citrate is still the first-line therapy for ovulation induction. ${ }^{13}$ In their study of 570 ovulation induction cycles in 150 women they did not find a difference in ovulation rates in clomiphene citrate (CC), tamoxifen and letrozole but pregnancy rates were higher in $\mathrm{CC}$ cycles and there is no added advantage of letrozole or tamoxifen over CC.

\section{Objectives}

Objective of current investigation was to study effect of melatonin supplementation to clomiphene citrate ovulation inductions cycles on ovulation and conception rates.

\section{METHODS}

This was a retrospective appraisal of infertility cases in which ovulation induction was planned with clomiphene citrate and melatonin. We included women with primary and secondary infertility in women with anovulatory infertility, unexplained infertility and endometriosis, over two-year duration January 2017 till December 2019 at Shri Guru Ram Rai Institute of Medical and Health Sciences, Dehradun. We excluded women with tubal factor and male factor infertility.

The study included 52 women with anovulatory, unexplained infertility and women with endometriosis related infertility, with 69 cumulative ovulation induction cycles. After taking complete history and a thorough examination, these women were investigated. Women were subjected to routine and special test, with $\mathrm{AMH}$, serum prolactin, thyroid profile, fasting blood sugar, fasting plasma insulin, serum LH and FSH was done on day two of a natural or progesterone induced menstrual 
cycle. Semen analysis was done with the usual three-day abstinence.

All women received a combination of clomiphene citrate $(50 \mathrm{mg})$ and melatonin $(3 \mathrm{mg})$ from day two till day six of the menstrual cycle (or induced cycle), staring with 50 $\mathrm{mg} \mathrm{CC}$ and increasing to $100 \mathrm{mg}$ and $150 \mathrm{mg}$. in subsequent cycles if there was no ovulation. The tablets were given at bedtime to reduce drug induced sleepiness. Follicular monitoring was done by TVS from day nine onwards and follicular growth and endometrial thickness was noted. An HCG trigger was given when dominant follicle attained size of $18-22 \mathrm{~mm}$ and an endometrial thickness more than $6 \mathrm{~mm}$. Rate of ovulation and conception per cycle was noted and analysed.

Table 3: Pregnancy outcome.

\begin{tabular}{|lll|}
\hline Pregnancy outcome & $\mathbf{N}$ & $\%$ \\
\hline Normal delivery & 15 & 36.5 \\
\hline LSCS & 11 & 11.26 \\
\hline Missed abortion & 2 & 4.8 \\
\hline Ongoing pregnancy & 13 & 31.7 \\
\hline
\end{tabular}

\section{RESULTS}

A total of 52 cases were analysed retrospectively, with 69 cumulative ovulation induction cycles using a combination of clomiphene citrate and melatonin. These cases were similar in their demographic profile. The ages ranged from 22 to-37 years (Table 1). There were 31 $(59.6 \%)$ cases of primary infertility and $21(40.21 \%)$ cases with secondary infertility. Out of these 52 cases, majority, 24 (46\%) were anovulatory infertility, 19(36\%) were unexplained infertility, $3(5.7 \%)$ had a previous ectopic, 4 (7.6\%) women were diagnosed cases of PID (with at least one tube patent) and $3(3.8 \%)$ had endometriosis (Table 2).

Table 1: Age group distribution.

\begin{tabular}{|l|l|l|}
\hline Age group (years) & $\mathbf{N}$ & $\%$ \\
\hline $\mathbf{2 0 - 2 5}$ & 16 & 30.7 \\
\hline $\mathbf{2 6 - 3 0}$ & 18 & 34 \\
\hline $\mathbf{3 1 - 3 5}$ & 13 & 25 \\
\hline $\mathbf{3 5}$ & 5 & 9.6 \\
\hline
\end{tabular}

Table 2: Cause of infertility.

\begin{tabular}{|lll|}
\hline Cause of infertility & N & $\%$ \\
\hline PCOS/anovulation & 24 & 46 \\
\hline Unexplained & 19 & 36 \\
\hline $\begin{array}{l}\text { PID } \\
\text { (at least one tube patent) }\end{array}$ & 4 & 7.6 \\
\hline Endometriosis & 2 & 3.8 \\
\hline Previous ectopic pregnancy & 3 & 5.6 \\
\hline
\end{tabular}

The acceptability and feasibility of induction protocol was universal, we saw $77.9 \%$ ovulation rates and $82 \%$ conception rates with $\mathrm{CC}$ and melatonin regimen for ovulation induction. There were 41 pregnancies out of 50 ovulations in 69 cumulative induction cycles. There were minimal side effects and some women complained of giddiness (6\%), and vaginal dryness (10.6\%). Most women had no side effects and the drug dose and schedule was acceptable to all. In all women- except two cases-pregnancy was continued to term without complication and resulted in healthy live births. There were $5(12.1 \%)$ cases of gestational diabetes, controlled with metformin and about 4 (9.7) women developed preeclampsia which an incidence similar to the overall incidence of preeclampsia. Pregnancy outcome (Table 3) was favourable in 25 women with normal delivery in 15 $(36.6 \%)$ cases and caesarean section in $11(11.6 \%)$ cases, and 13 pregnancies $(31.7 \%)$ were ongoing at the time of study. There were no perinatal complications. Two women had missed abortion and needed termination of pregnancy. In our series all pregnancies were singleton pregnancy and there was no multiple pregnancy which is a common effect of multiple ovulations with $\mathrm{CC}$ inductions.

\section{DISCUSSION}

Oocyte quality is single most important factor in determining successful conception in infertile women undergoing ART. ${ }^{14}$ Oocyte quality often dictates reproductive outcome. The cytoskeleton integrity of gametes and mitochondrial function has an important role in chromosomal spindle formation and consequently of a healthy euploid embryo necessary for conception. ${ }^{14}$ Many factors affect oocyte quality ranging from, age, obesity, cause of infertility, co-morbid conditions and some male factors, like sperm DNA fragmentation. ${ }^{14}$ Aging women have reduced fertility rates due to declining oocyte quality directly proportional to the level of oxidative stress experienced by the women of higher age. A 29year-old woman has about a $40 \%$ chance of achieving pregnancy during an ART cycle whereas this is reduced to $32 \%$ and $0 \%$ at 36 years and 46 years respectively. ${ }^{15-16}$ Most women who benefited from treatment in our study belonged to $26-30(34 \%)$ and $31-35(25 \%)$ years age group. Oxidative stress affects both male and female gametes and is an undervalued cause which has a potential reduce oocyte quality, in infertility management, as has been shown by a large double blind randomised trial by Shavietal. ${ }^{1}$ Recently there has been a great emphasis on use of antioxidants as adjuvants in ART cycle to improve oocyte quality. ${ }^{17}$ There are studies that show composition of follicular fluid affects oocyte maturation and high concentration of myo-inositol and melatonin in follicular fluid is seen to be important for oocyte maturation. ${ }^{18}$ Melatonin works as an antioxidant to reduces intracellular ROSs helping in oocyte maturation and may have role in other cellular processes. 
Recent studies highlight the important of the follicular fluid (FF) in oocyte maturation. Especially, high concentration of two different compounds such as myoinositol (MI) and melatonin was shown to be essential for optimal oocyte maturation. ${ }^{18}$ Melatonin is natural hormone secreted by pineal gland and plays an important role in minimising ROC from gametes, reducing stress, thereby aiding the conception success. We, with a view to improve pregnancy rates in clomiphene citrate ovulation induction in our study observed a significant benefit of addition of melatonin to the clomiphene citrate ovulation induction programs in improving conception rates. In the present study out of 52 infertile women who were given ovulation induction with $\mathrm{CC}$ and melatonin in 69 cumulative ovulation induction cycles, 50 women $(77.9 \%)$ had ovulation out of which 41 $(59.4 \%)$ women had successful conception. Although our ovulation rates with clomiphene citrate agree with rates quoted in the literature our conception rates were higher. Usadi $\mathrm{R}$ et al in their study of clomiphene citrate ovulation induction observed a $40 \%$ conception rate and in our the conception rate was higher at $59.4 \% .{ }^{12}$ In order to achieve high quality oocytes Giovanni et al used myoinositol and melatonin and saw that is significantly improved quality of oocyte and embryo. ${ }^{14}$ In a systematic review and meta-analysis of randomised controlled trials Kai-Lun Hu colleagues observed that melatonin treatment increases clinical pregnancy rates and it also increases number of good quality mature oocyte collected, and good quality embryos. ${ }^{19}$ This study also concluded that melatonin does not improve live birth rate which was contrary to our results, since we had good pregnancy outcomes, with only two miscarriages and no instance of multiple births. The observation by Shavi et al in their RCT found no extra benefit of melatonin addition. A similar conclusion was drawn by Ludmila et al in a systematic review and meta-analysis of RCTs on melatonin supplementation for controlled ovarian stimulation (COS) which stated that there was uncertainty about use of melatonin addition in ART cycle and larger studies are needed before clinical applications can be recommended. ${ }^{20}$ They observed that melatonin addition in COS does not reduce the chance of clinical pregnancy and number of oocytes retrieved. Although our study was smaller, the results were encouraging and we found supplementation of melatonin with $\mathrm{CC}$ for ovulation induction beneficial in improving conception and live birth rates.

\section{Limitations}

Limitation of current study was it was a small study but the results were encouraging. May be larger studies with more cases will determine actual benefit of melatonin in infertility management.

\section{CONCLUSION}

Oxidative stress is an unappreciated condition that can reduce the oocyte quality and affect conception rates in women undergoing infertility treatment. Melatonin, as an anti-oxidant may improve, conception and live birth rates when added to clomiphene citrate ovulation induction protocols.

\section{Funding: No funding sources \\ Conflict of interest: None declared}

Ethical approval: The study was approved by the Institutional Ethics Committee

\section{REFERENCES}

1. Shavi F, Luk R. Melatonin: shedding light on infertility? - a review of the recent literature. J Ovarian Res. 2014;7:98.

2. Walczak-Jedrzejowska R, Wolski JK, SlowikowskaHilczer J. The role of oxidative stress and antioxidants in male fertility. Cent European J Urol. 2013;66(1): 60-7.

3. Sindhia V, Guptaa V, Sharma S, Kumaria BR, Neeti D. Potential applications of antioxidants - A review. J Pharm Res. 2013;7(9):828-83.

4. Tamura H, Takasaki A, Taketani T, Tanabe M, Kizuka F, Lee L, et al. The role of melatonin as an antioxidant in the follicle. J Ovarian Res. 2012;5:5.

5. Partonen T. Melatonin-dependent infertility. Med Hypotheses. 1999;52(5):487-8.

6. Brzezinski A, Seibel MM, Lynch HJ, Deng MH, Wurtman RJ. Melatonin in human preovulatory follicular fluid. J Clin Endocrinol Metab. 1987; 64(4):865-7.

7. Poeggeler B, Reiter RJ, Tan DX, Chen LD, Manchester LC. Melatonin, hydroxyl radicalmediated oxidative damage, and aging: a hypothesis. J Pineal Res. 1993;14(4):151-68.

8. Rahman K. Studies on free radicals, antioxidants, and co-factors. Clin Interv Aging. 2007;2:219-36

9. Fernando S, Osianlis T, Vollenhoven B. A pilot double-blind randomised placebo-controlled doseresponse trial assessing the effects of melatonin on infertility treatment (MIART): study protocol. BMJ. 2014;4:e005986.

10. Zeng H, Ren Z, Yeung W. Low mitochondrial DNA and ATP contents contribute to the absence of birefringent spindle imaged with PolScope in in vitro matured human oocytes. Hum Reprod. 2007; 22:1681-6.

11. Song C, Peng W, Yin S, Zhao J, Fu B, Zhang J, et al. Melatonin improves age-induced fertility decline and attenuates ovarian mitochondrial oxidative stress in mice. Sci Rep. 2016 Oct 12;6:35165.

12. Usadi R, Fritz M. Induction of ovulation with clomiphene citrate. Glob Libr Women's Med. 2008;23:512-9.

13. Seyedoshohadaei F, Zandvakily F, Shahgeibi S. Comparison of the effectiveness of clomiphene citrate, tamoxifen and letrozole in ovulation induction in infertility due to isolated unovulation. Iran $\mathrm{J}$ Reprod Med. 2012;10(6):531-6. 
14. Salvatore Giovanni Vitale, Paola Rossetti, Francesco Corrado, Agnese Maria Chiara Rapisarda, Sandro La Vignera, Rosita Angela Condorelli, Gaetano Valenti, Fabrizio Sapia, Antonio Simone Laganà, and Massimo Buscema How to Achieve High-Quality Oocytes? The Key Role of Myo-Inositol and Melatonin. Int J Endocrinol. 2016; 2016: 4987436

15. Vittorio U, Vitale SG, Rossetti P, Corrado F. How to Achieve High-Quality Oocytes? The Key Role of Myo-Inositol and Melatonin. Int $\mathrm{J}$ Endocrinol. 2016;2016:4987436.

16. Leridon H. Can assisted reproduction technology compensate for the natural decline in fertility with age? A model assessment. Hum Reprod. 2004; 19(7):1548-53.

17. Wiener-Megnazi Z, Vardi L, Lissak A, Shnizer S, Reznick AZ, Ishai D, et al. Oxidative stress indices in follicular fluid as measured by the thermochemiluminescence assay correlate with outcome parameters in in vitro fertilization. Fertil Steril. 2004;82(3):1171-6.
18. Berridge MJ. Inositol lipids and cell proliferation. Biochim Biophys Acta. 1987;907(1):33-45.

19. Hu KL, Ye X, Wang S, Zhang D. Melatonin Application in Assisted Reproductive Technology: A systematic review and meta-analysis of randomized trials. 2020;11:333.

20. Ludmila MD, Seko, Raphael M, Moroni V, et al. Melatonin supplementation during controlled ovarian stimulation for women undergoing assisted fertility technology: A systematic and review and Metaanalysis of randomised controlled trials. Fertil Steril. 2014;101(1):282-84.

Cite this article as: Chaudhary A, Tanwar M, Agarwal A, Singh P, Negi P. Effect of melatonin addition in ovulation induction protocols with clomiphene citrate in management of infertility. Int $\mathbf{J}$ Reprod Contracept Obstet Gynecol 2021;10:4438-42. 